\title{
Singularities of Optimal Control Problems on some Six Dimensional Lie groups
}

\author{
James Biggs, William Holderbaum, and Velimir Jurdjevic
}

\begin{abstract}
This paper considers the motion planning problem for oriented vehicles travelling at unit speed in a threedimensional space. A Lie group formulation arises naturally and the vehicles are modelled as kinematic control systems with drift defined on the orthonormal frame bundles of particular Riemannian manifolds, specifically the three-dimensional space forms Euclidean space $\mathbb{E}^{3}$, the sphere $\mathbb{S}^{3}$ and the hyperboloid $\mathbb{H}^{3}$. The corresponding frame bundles are equal to the Euclidean group of motions $S E(3)$, the rotation group $S O(4)$ and the Lorentz group $S O(1,3)$. The Maximum Principle of optimal control, shifts the emphasis for these systems to the associated Hamiltonian formalism. For an integrable case the extremal curves are explicitly expressed in terms of elliptic functions. In this paper, a study at the singularities of the extremal curves are given, which correspond to critical points of these elliptic functions. The extremal curves are characterized as the intersections of invariant surfaces and are illustrated graphically at the singular points. It is then shown that the projections of the extremals onto the base space, called elastica, at these singular points, are curves of constant curvature and torsion, which in turn implies that the oriented vehicles trace helices.
\end{abstract}

Index Terms-Optimal Control, Lie Groups, Integrable Hamiltonian Systems, Singularities.

\section{INTRODUCTION}

This paper is motivated by the problem of motion planning for oriented vehicles moving with unit speed in a three-dimensional space, such as the airplane landing problem [1]. For such problems the orientation of the vehicle is naturally represented by an orthonormal frame over a point in the underlying manifold, that is, the configuration space of the vehicle can be taken as the orthonormal frame bundle of the manifold, and the motions of the vehicle are described by curves in this bundle. In this paper we consider three-dimensional spaces for which the orthonormal frame bundle coincides with the isometry group. They are, the Euclidean space $\mathbb{E}^{3}$, the sphere $\mathbb{S}^{3}$ and the hyperboloid $\mathbb{H}^{3}$. The corresponding isometry groups are the Euclidean group of motion $S E(3)$, the rotation group $S O(4)$ and the Lorentzian group $S O(1,3)$. The Euclidean setting has been used to study multi-vehicle formation control of Unmanned air vehicles [2], the airplane landing problem [1] and the control of underactuated Underwater Vehicles [3]. In each of these cases the oriented vehicles trace paths in $\mathbb{E}^{3}$. In this paper we generalize the Euclidean frame, simultaneously

J. Biggs is with School of Systems Engineering, University of Reading, Reading, United Kingdom j.biggs@rdg.ac.uk

W. Holderbaum is with School of Systems Engineering, University of Reading, Reading, United Kingdom w.holderbaumerdg.ac.uk

V. Jurdjevic is with the Department of Mathematics, University of Toronto, Toronto, Canada jurdjemath.toronto.edu studying oriented vehicles in the space forms $\mathbb{E}^{3}, \mathbb{S}^{3}$ and $\mathbb{H}^{3}$, as in [4].

In [2] the authors use the Euclidean Serret-Frenet Frame to model Unmanned Air vehicles as particles moving at unit speed in Euclidean space, where the controls take the form of the geometric invariants curvature and torsion. In addition [5] uses a generalized Serret-Frenet frame adapted to curved spaces to describe the motion of relativistic particles in $\mathbb{S}^{3}$ and $\mathbb{H}^{3}$. For a description of the Serret-Frenet frame and its formulation on Lie groups, see [6]. Although the SerretFrame is adequate in describing the motions of particles it is inadequate in describing oriented bodies.

More general Euclidean frames have been used to describe the motion of underwater vehicles in [3] and airplanes in [1]. In this work we use the most general orthonormal frames to simultaneously study the motion planning problem for oriented vehicles travelling in $\mathbb{E}^{3}, \mathbb{S}^{3}$ and $\mathbb{H}^{3}$. For a detailed description of space forms, their frame bundles and their tangent spaces see [7].

The motion planning problem for oriented vehicles has been tackled using local representations of Lie groups i.e. the Wei-Norman representation and Magnus representation to describe the behavior of such systems and then classical methods from nonlinear control e.g. averaging on $\mathbb{R}^{n}$ have been adapted to these coordinate representations, see [8]. However, because in general these representations are local, only small reorientations can be performed at any one time and highlights the need for global methods to plan larger, more energy efficient manoeuvres.

The global motion planning problem for systems evolving on Lie groups has been addressed using optimal control theory. In [9] the authors derive control functions such that the resulting trajectory in the configuration space interpolates a given set of points, subject to some physically interesting cost function. The optimal control problem for underactuated kinematic systems on Riemannian manifolds, known as sub-Riemannian optimal control problems, have been studied in [8], [10] and optimal control of underactuated dynamic systems in [11]. In [1] the authors use the Maximum Principle of optimal control to design landing trajectories for airplanes. In this paper we propose to tackle the global motion planning problem for oriented vehicles travelling at constant speed in a three-dimensional space using the coordinate free Maximum Principle of optimal control, see [12] and [6]. The advantage of using the Maximum Principle to solve the motion planning problem is that not only do we steer the vehicle from point to point but do so while minimizing some practical cost function. 
The application of the Maximum Principle of optimal control shifts the emphasis to the language of symplectic geometry and to the associated Hamiltonian formalism. The Maximum principle states that the optimal solutions are the projections of the extremal curves onto the base manifold, where the extremal curves are solutions of certain Hamiltonian systems on the cotangent bundle $T^{*} G$. As the base manifolds of the oriented vehicle is a Lie group $G$, the cotangent bundle $T^{*} G$ can be realized as the direct product $G \times \mathfrak{g}^{*}$ where $\mathfrak{g}^{*}$ is the dual of the Lie algebra $\mathfrak{g}$ of $G$. Therefore, the original Hamiltonian defined on $T^{*} G$ can be written as a reduced Hamiltonian on the dual of the Lie algebra $\mathfrak{g}^{*}$. The corresponding Hamiltonian vector fields are then expressed in non-canonical form and will be referred to as the reduced Hamiltonian vector fields.

For a symmetric oriented vehicle, where two moments of inertia $c_{2}$ and $c_{3}$ are equal, the Hamiltonian vector fields are integrable (see [13] for a detailed description of integrable Hamiltonian systems on Lie groups) and we derive explicit expressions for the extremal curves. For integrable systems their topological and qualitative properties such as bifurcations and singularities are of great importance and knowledge about these properties will give us some information about the dynamical properties of a perturbed Hamiltonian or near integrable Hamiltonian, as outlined in [14].

For the reduced integrable Hamiltonian system the extremal curves are explicitly expressed by elliptic functions. Using these explicit expressions we investigate the singularities of the Hamiltonian vector fields. The singularities, or singular points, are equilibria for the reduced Hamiltonian system and are defined at the roots of the cubic function that appear in the explicit expression of the extremal curves. The singularities of the reduced Hamiltonian are important in the motion control of vehicles as they coincide with relative equilibria for the original Hamiltonian system. Indeed, it is shown that the projections of the extremal curves at a singularity onto the base space are helical curves. This implies that the extremal controls at a singularity induce steady motions of the vehicle i.e. constant translation and/or constant rotation. Once these relative equilibria have been identified such techniques for stabilization and control can be used as in [8]. Studying these systems and their singularities provide insight into the rich qualitative and topological nature inherent in the motion planning of vehicles.

In this paper the motions of oriented vehicles are restricted to move at unit speed. Under this restriction the motion planning problem of steering the vehicle from an initial configuration to a final configuration, in a fixed time, while minimizing the amount of manoeuvering the vehicle will do, can be equated to the elastic rod problem of Kirchhoff, which is detailed in [13]. In the elastic problem the projections of the extremal curves onto the base space, called elastic curves, reflect the shape of an elastic rod, of fixed length, forced to have some initial and final position and orientation. Therefore, in this motion planning problem, the oriented vehicles will trace elastic curves. In the Euclidean case, Kirchhoff elastic rods have been used to model practical problems such as the dynamic formation of DNA and to explain the looping of marine cables, see [15].

The original contributions in this paper are summarized in the following statement:

\section{StATEMEnt OF CONTRibutions}

- The extremals are explicitly expressed in terms of cubic curves which are parameterized by elliptic functions.

- The curvature and torsion of the elastic curves are explicitly expressed by elliptic functions.

- The critical points of this cubic correspond to periodic extremal curves characterized as the intersections of invariant surfaces, which are illustrated graphically.

- The elastic curves at the singularity are shown to be of constant curvature and constant torsion and therefore the oriented vehicles trace helices.

\section{EXTREMAL CURVES IN $\mathfrak{s e}(3)^{*}, \mathfrak{s o}(4)^{*}$ AND $\mathfrak{s o}(1,3)^{*}$}

In this section we will state the elastic problem and equate it to the motion planning problem for oriented vehicles moving with unit speed in a three-dimensional space. Firstly, the group $G$ is used to represent the frame bundle of the space forms. The analysis here is restricted to the three dimensional space forms and therefore the corresponding frame bundles are the matrix Lie groups $S E(3), S O(4)$ and $S O(1,3)$. We identify $T G$ with $G \times \mathfrak{g}$ where $\mathfrak{g}$ is the Lie algebra of $G$ via the left translations. The elastic problem on the three dimensional space forms concerns the solutions $g(t) \in G$ of the leftinvariant differential system:

$$
\begin{aligned}
& \frac{d g}{d t}(t)=g(t)\left(B_{1}+\sum_{i=1}^{3} u_{i} A_{i}\right) \\
& =g(t)\left(\begin{array}{cccc}
0 & -\varepsilon & 0 & 0 \\
1 & 0 & -u_{3} & u_{2} \\
0 & u_{3} & 0 & -u_{1} \\
0 & -u_{2} & u_{1} & 0
\end{array}\right)
\end{aligned}
$$

that minimize the expression

$$
\frac{1}{2} \int_{0}^{T}(u(t), Q u(t)) d t
$$

subject to the given boundary condition $g(0)=g_{0}, g(T)=g_{1}$. $g(t) \in G$ where $G$ depends on $\varepsilon$ and is $S E(3)$ for $\varepsilon=0, S O(4)$ for $\varepsilon=1$ and $S O(1,3)$ for $\varepsilon=-1$, so we are simultaneously studying all three cases. $B_{1}, A_{1} \ldots, A_{3}$ are given matrices in the Lie algebra $\mathfrak{g}$ of $G$, the $u_{i}$ 's play the role of the control functions and $Q$ is a positive definite $3 \times 3$ matrix. This problem can be identified with the elastic rod problem of Kirchhoff, by replacing time $t$ with the arc length parameter of the curve $s$ and in the expression (2) the terminal time $T$ should be replaced with the length of the rod $l$. Then $\gamma(s)$ corresponds to the central line of the rod. The physical characteristics of the rod, related to the geometric shape of its cross section are reflected in the constants, call $c_{i}$, dependent on the positive definite $3 \times 3$ matrix $Q$. The control functions represent the strains of the elastic rod. Then (1) describes the frame deformations of the rod on the frame bundle $G$ of the 
space form $M$. It follows that the problem of minimizing the expression (2) is equivalent to minimizing locally the amount of bending and twisting in the rod.

Proceeding to equate the elastic problem to the problem of motion planning of a vehicle, the principal moments of inertia $c_{i}$ of the vehicle are dependent on the matrix $Q$ and the controls $u_{i}$ relate to the angular velocity of the vehicle. It follows that (1) describe the kinematic equations of the vehicle such that the vehicle traces out a trajectory $\gamma(t) \in M$ which are related to $g(t) \in G$ via the projection $\gamma(t)=g(t) \vec{e}_{1}$ where $\vec{e}_{1}$ is a basis element in a standard orthonormal frame $\vec{e}_{1}, \vec{e}_{2}, \vec{e}_{3}, \vec{e}_{4} \in \mathbb{R}^{4}$. The projected curves $\gamma(t) \in M$ are called elastic curves, where $M=\mathbb{E}^{3}$ when $g(t) \in S E(3), M=\mathbb{S}^{3}$ when $g(t) \in S O(4)$ and $M=\mathbb{H}^{3}$ when $g(t) \in S O(1,3)$. It follows from (1) that the vehicle is restricted to travel at unit speed $\left\|\frac{d \gamma(t)}{d t}\right\|=1$ and in addition $\frac{d \gamma(t)}{d t}$ coincides with the first leg of the frame (see [7] for detail). The solutions $g(t) \in G$ of (1) while minimizing the expression (2) are locally optimal, that is optimal for small terminal time $T$, however as the terminal time grows they may stop being optimal. For simplicity of terminology we will refer to all projections as the optimal solutions even though the nature of cut-locus and conjugate points have not been considered, see [6]. Minimizing the cost function (2) is then equivalent to minimizing the control energy of the vehicle between an initial position and orientation and a final position and orientation.

The Maximum Principle of optimal control identifies the appropriate left-invariant Hamiltonian $H$ on the dual of the Lie algebra $\mathfrak{g}^{*}$, (see [6]). The Maximum Principle, as a general necessary condition of optimality, leads to the appropriate Hamiltonian on the cotangent bundle of the Lie Group $G$, hence the extremal curves belong to $T^{*} G$. Then the Maximum Principle considers the lift of the optimization problem to the cotangent manifold $T^{*} G$. The control Hamiltonian is written as:

$$
H(p, u)=p\left(g B_{1}\right)+\sum_{i=1}^{3} u_{i} p\left(g A_{i}\right)-p_{0}\left(\frac{1}{2} \sum_{i=1}^{3} c_{i} u_{i}^{2}\right)
$$

where $p \in T_{g}^{*} G$ and $p_{0} \geqslant 0$ is a fixed positive constant and $H(p, u)$ is a concave function with respect to $u_{i}$. It then follows from the Maximum Principle that the extremal control functions $u_{i}^{*}$ are determined from the condition:

$$
\frac{d H}{d u_{i}}=0
$$

differentiating (3) with respect to $u_{i}$ gives

$$
\frac{d H}{d u_{i}}=p\left(g A_{i}\right)-c_{i} u_{i}
$$

where $i=1,2,3$. Therefore, the extremal controls are given in feedback form:

$$
u_{i}^{*}=\frac{1}{c_{i}} p\left(g A_{i}\right)
$$

where $i=1,2,3$. Because of the non-holonomic nature of these problems, the extremal curves that correspond to an optimal trajectory can be either abnormal or normal i.e. there are two types of Hamiltonian to consider. $p_{0}$ is set to 1 for normal extremals and 0 for abnormal extremals. All of these problems admit abnormal extremals, however, because of the regularity of these variational problems each optimal trajectory is a projection of a regular extremal curve. Therefore, assume $p_{0}=1$ to consider only regular extremals.

The Hamiltonian for such systems are functions on the cotangent bundle $T^{*} G$ which can be trivialized from the left such that $T^{*} G=G \times \mathfrak{g}^{*}$. Therefore, the appropriate Hamiltonian is a function on $\mathfrak{g}^{*}$ the dual of the Lie algebra $\mathfrak{g}$ of $G$. The Hamiltonian (3) can be pulled back by the left or right. The pull-back in this case is explicitly stated as $p(\cdot)=\hat{p}\left(g^{-1}(\cdot)\right)$. i.e $p \in T^{*} G$ is pulled back to give a function $\hat{p} \in \mathfrak{g}^{*}$. The control Hamiltonian can then be written as

$$
H(\hat{p}, u)=\hat{p}\left(B_{1}\right)+\sum_{i=1}^{3} u_{i} \hat{p}\left(A_{i}\right)-\frac{1}{2} \sum_{i=1}^{3} c_{i} u_{i}^{2}
$$

This Hamiltonian is a function of the controls only, and so it does not depend explicitly on elements in $G$. In other words it is left invariant in that it does not change by the group multiplication on the left. In addition, define the extremal curves $p_{i}=\hat{p}\left(B_{i}\right)$ and $M_{i}=\hat{p}\left(A_{i}\right)$ then it follows that the extremal controls can be expressed as

$$
u_{i}^{*}=\frac{1}{c_{i}} M_{i}
$$

it follows from the formula (5) that the extremals $M_{i}$ correspond to components of angular momentum. Substituting these back into (4) gives the optimal Hamiltonian

$$
H^{*}=p_{1}+\frac{1}{2}\left(\frac{M_{1}^{2}}{c_{1}}+\frac{M_{2}^{2}}{c_{2}}+\frac{M_{3}^{2}}{c_{3}}\right)
$$

the extremal control functions $u_{i}{ }^{*}$ are additionally substituted into (1) to yield

$$
g^{-1} \frac{d g}{d t}=\left(\begin{array}{cccc}
0 & -\varepsilon & 0 & 0 \\
1 & 0 & -M_{3} / c_{3} & M_{2} / c_{2} \\
0 & M_{3} / c_{3} & 0 & -M_{1} / c_{1} \\
0 & -M_{2} / c_{2} & M_{1} / c_{1} & 0
\end{array}\right)
$$

To proceed it is essential to recognize some geometric facts about these Lie algebras. The variational problem on Lie groups in this paper are associated with the Cartan decomposition of $\mathfrak{g}$ of a Lie group $G$ into the factors $\mathfrak{p}$ and $\mathfrak{k}$ which satisfy the classic relations

$[\mathfrak{k}, \mathfrak{k}] \subseteq \mathfrak{k},[\mathfrak{p}, \mathfrak{k}] \subseteq \mathfrak{p}$ and $[\mathfrak{p}, \mathfrak{p}] \subseteq \mathfrak{k}$

where $\mathfrak{k}$ consists of all matrices of the form

$$
\left(\begin{array}{cccc}
0 & 0 & 0 & 0 \\
0 & 0 & -\alpha_{3} & \alpha_{2} \\
0 & \alpha_{3} & 0 & -\alpha_{1} \\
0 & -\alpha_{2} & \alpha_{1} & 0
\end{array}\right)
$$

and $\mathfrak{p}$ consists of the matrices

$$
\left(\begin{array}{cccc}
0 & -\varepsilon b_{1} & -\varepsilon b_{2} & -\varepsilon b_{3} \\
b_{1} & 0 & 0 & 0 \\
b_{2} & 0 & 0 & 0 \\
b_{3} & 0 & 0 & 0
\end{array}\right)
$$


The corresponding basis elements for $\mathfrak{k}$ and $\mathfrak{p}$ are:

$$
\begin{aligned}
& A_{1}=\left(\begin{array}{cccc}
0 & 0 & 0 & 0 \\
0 & 0 & 0 & 0 \\
0 & 0 & 0 & -1 \\
0 & 0 & 1 & 0
\end{array}\right), A_{2}=\left(\begin{array}{cccc}
0 & 0 & 0 & 0 \\
0 & 0 & 0 & 1 \\
0 & 0 & 0 & 0 \\
0 & -1 & 0 & 0
\end{array}\right) \\
& A_{3}=\left(\begin{array}{cccc}
0 & 0 & 0 & 0 \\
0 & 0 & -1 & 0 \\
0 & 1 & 0 & 0 \\
0 & 0 & 0 & 0
\end{array}\right), B_{1}=\left(\begin{array}{cccc}
0 & -\varepsilon & 0 & 0 \\
1 & 0 & 0 & 0 \\
0 & 0 & 0 & 0 \\
0 & 0 & 0 & 0
\end{array}\right) \\
& B_{2}=\left(\begin{array}{cccc}
0 & 0 & -\varepsilon & 0 \\
0 & 0 & 0 & 0 \\
1 & 0 & 0 & 0 \\
0 & 0 & 0 & 0
\end{array}\right), B_{3}=\left(\begin{array}{cccc}
0 & 0 & 0 & -\varepsilon \\
0 & 0 & 0 & 0 \\
0 & 0 & 0 & 0 \\
1 & 0 & 0 & 0
\end{array}\right)
\end{aligned}
$$

$A_{1}, A_{2}, A_{3}, B_{1}, B_{2}, B_{3}$ describe infinitesimal motion in the roll, pitch, yaw, surge, sway and heave directions of the vehicle respectively. The Lie bracket is defined as $[X, Y]=X Y-Y X$ and the corresponding Lie bracket table is then:

\begin{tabular}{|c|c|c|c|c|c|c|}
\hline$[]$, & $A_{1}$ & $A_{2}$ & $A_{3}$ & $B_{1}$ & $B_{2}$ & $B_{3}$ \\
\hline$A_{1}$ & 0 & $A_{3}$ & $-A_{2}$ & 0 & $B_{3}$ & $-B_{2}$ \\
$A_{2}$ & $-A_{3}$ & 0 & $A_{1}$ & $-B_{3}$ & 0 & $B_{1}$ \\
$A_{3}$ & $A_{2}$ & $-A_{1}$ & 0 & $B_{2}$ & $-B_{1}$ & 0 \\
$B_{1}$ & 0 & $B_{3}$ & $-B_{2}$ & 0 & $\varepsilon A_{3}$ & $-\varepsilon A_{2}$ \\
$B_{2}$ & $-B_{3}$ & 0 & $B_{1}$ & $-\varepsilon A_{3}$ & 0 & $\varepsilon A_{1}$ \\
$B_{3}$ & $B_{2}$ & $-B_{1}$ & 0 & $\varepsilon A_{2}$ & $-\varepsilon A_{1}$ & 0 \\
\hline
\end{tabular}

Using the optimal Hamiltonian (6), it is possible to construct the corresponding Hamiltonian vector fields $X_{H^{*}}$ using the Poisson bracket defined on the symplectic manifold. The Hamiltonian vector fields are calculated using the formula $X_{H^{*}}[\cdot]=\left\{\cdot, H^{*}\right\}$ where the Poisson bracket is associated with the Lie bracket by $\left\{M_{i}, M_{j}\right\}=-\hat{p}\left(\left[A_{i}, A_{j}\right]\right)$. Therefore, it follows that:

$$
\begin{aligned}
& \frac{d M_{1}}{d t}=\left\{M_{1}, H^{*}\right\}=\left\{M_{1}, p_{1}\right\}+\frac{M_{1}}{c_{1}}\left\{M_{1}, M_{1}\right\} \\
& +\frac{M_{2}}{c_{2}}\left\{M_{1}, M_{2}\right\}+\frac{M_{3}}{c_{3}}\left\{M_{1}, M_{3}\right\} \\
& =0+0-\frac{1}{c_{2}} M_{2} M_{3}+\frac{1}{c_{3}} M_{3} M_{2} \\
& =\frac{c_{2}-c_{3}}{c_{2} c_{3}} M_{2} M_{3}
\end{aligned}
$$

the remaining derivations of the Hamiltonian vector fields are left to the reader and yield:

$$
\left\{\begin{array}{l}
\frac{d M_{1}}{d t}=\left\{M_{1}, H^{*}\right\}=\frac{-M_{2} M_{3}}{c_{2}}+\frac{M_{2} M_{3}}{c_{3}} \\
\frac{d M_{2}}{d t}=\left\{M_{2}, H^{*}\right\}=\frac{M_{1} M_{3}}{c_{1}}-\frac{M_{1} M_{3}}{c_{3}}+p_{3} \\
\frac{d M_{3}}{d t}=\left\{M_{3}, H^{*}\right\}=\frac{-M_{1} M_{2}}{c_{1}}+\frac{M_{1} M_{2}}{c_{2}}-p_{2} \\
\frac{d p_{1}}{d t}=\left\{p_{1}, H^{*}\right\}=\frac{-M_{2} p_{3}}{c_{2}}+\frac{p_{2} M_{3}}{c_{3}} \\
\frac{d p_{2}}{d t}=\left\{p_{2}, H^{*}\right\}=\frac{M_{1} p_{3}}{c_{1}}-\frac{p_{1} M_{3}}{c_{3}}+\varepsilon M_{3} \\
\frac{d p_{3}}{d t}=\left\{p_{3}, H^{*}\right\}=-\frac{M_{1} p_{2}}{c_{1}}+\frac{p_{1} M_{2}}{c_{2}}-\varepsilon M_{2}
\end{array}\right.
$$

In this paper the analysis is restricted to an integrable case of the Hamiltonian vector fields $X_{H^{*}}$. Explicitly a Hamiltonian function on a symplectic manifold $N$ of dimension $2 n$ is said to be integrable if there exist functions $\varphi_{2}, \ldots, \varphi_{n}$ on $N$ that together with the Hamiltonian $H^{*}=\varphi_{1}$ satisfy the following two properties:

- $\varphi_{1}, \ldots, \varphi_{n}$ are functionally independent i.e the differentials $d \varphi_{1}, \ldots, d \varphi_{n}$ are linearly independent for an open subset of $N$.

- The functions $\varphi_{1}, \ldots, \varphi_{n}$ are in involution.

Thus, in identifying the $(n-1)$ functions $\varphi_{i}$ and the Hamiltonian function the system is completely integrable. In the mechanics literature these are called integrals of motion. The Casimir functions are constant on co-adjoint orbits of $G$, and are integrals of motion for any left-invariant Hamiltonian $H^{*}$. There are also two extra integrals of motion corresponding to their right-invariant Hamiltonian. They are in involution with each other, and also in involution with $H^{*}$ and the two Casimir functions. Hence, altogether they account for five independent integrals of motion and the system becomes completely integrable whenever there is just one more integral of motion. For left-invariant control systems defined on semisimple Lie algebras, the Casimir functions are derived through the invariance of the Killing form. In the case of $S E(3)$ the Killing form is degenerate and therefore the Casimir functions are derived in a different way (see [7] for a detailed description of their derivation), explicitly the Hamiltonian and Casimir functions are:

$$
\begin{gathered}
H^{*}=p_{1}+\frac{1}{2}\left(\frac{M_{1}^{2}}{c_{1}}+\frac{M_{2}^{2}}{c_{2}}+\frac{M_{3}^{2}}{c_{3}}\right) \\
I_{2}=p_{1}^{2}+p_{2}^{2}+p_{3}^{2}+\varepsilon\left(M_{1}^{2}+M_{2}^{2}+M_{3}^{2}\right) \\
I_{3}=p_{1} M_{1}+p_{2} M_{2}+p_{3} M_{3}
\end{gathered}
$$

An extra integral of motion exists in a case analogous to Lagrange's top [16]. Proceeding by equating $c_{2}=c_{3}$ and normalizing the constants such that $c_{2}=c_{3}=1$, and $c_{1} \neq 0$, yields an extra integral of motion required for Liouville integrability, see [16] for a description. The condition $c_{2}=c_{3}=1$ in (8) gives:

$$
\frac{d M_{1}}{d t}=0
$$

and therefore $M_{1}$ is a constant of motion which will be denoted $\sigma$, the Hamiltonian vector fields (8) reduce to:

$$
\left\{\begin{array}{l}
\frac{d M_{2}}{d t}=\frac{\sigma M_{3}}{c_{1}}-\sigma M_{3}+p_{3} \\
\frac{d M_{3}}{d t}=\frac{-\sigma M_{2}}{c_{1}}+\sigma M_{2}-p_{2} \\
\frac{d p_{1}}{d t}=-M_{2} p_{3}+p_{2} M_{3} \\
\frac{d p_{2}}{d t}=\frac{\sigma p_{3}}{c_{1}}-p_{1} M_{3}+\varepsilon M_{3} \\
\frac{d p_{3}}{d t}=-\frac{\sigma p_{2}}{c_{1}}+p_{1} M_{2}-\varepsilon M_{2}
\end{array}\right.
$$


In a similar manner to [1], we proceed to solve for the extremal control functions:

$$
\frac{d p_{1}}{d t}=p_{2} M_{3}-M_{2} p_{3}
$$

it follows that

$$
\left(\frac{d p_{1}}{d t}\right)^{2}=p_{2}^{2} M_{3}^{2}+p_{3}^{2} M_{2}^{2}-2 p_{2} p_{3} M_{2} M_{3}
$$

Using (9) and (10) write;

$$
\begin{aligned}
& 2\left(H^{*}-p_{1}\right)-\frac{\sigma^{2}}{c_{1}}=M_{2}^{2}+M_{3}^{2} \\
& I_{2}-p_{1}^{2}-\varepsilon\left(\sigma^{2}\right)=p_{2}^{2}+p_{3}^{2}+\varepsilon\left(M_{2}^{2}+M_{3}^{2}\right)
\end{aligned}
$$

multiplying the two equations in (14) gives:

$$
\begin{aligned}
& \left(I_{2}-p_{1}^{2}-\varepsilon\left(\sigma^{2}\right)\right)\left(2\left(H^{*}-p_{1}\right)-\frac{\sigma^{2}}{c_{1}}\right)= \\
& p_{2}^{2} M_{2}^{2}+p_{2}^{2} M_{3}^{2}+p_{3}^{2} M_{2}^{2}+p_{3}^{2} M_{3}^{2}+\varepsilon\left(\left(M_{2}^{2}+M_{3}^{2}\right)^{2}\right)
\end{aligned}
$$

To find explicit solutions it is necessary to use the Casimir function (11)

$$
I_{3}-p_{1} \sigma=p_{2} M_{2}+p_{3} M_{3}
$$

squaring (16) yields:

$$
\left(I_{3}-p_{1} \sigma\right)^{2}=p_{2}^{2} M_{2}^{2}+p_{3}^{2} M_{3}^{2}+2 p_{2} M_{2} p_{3} M_{3}
$$

Therefore, substituting (17) and (15) into (13) gives the following cubic function:

$$
\begin{aligned}
& f\left(p_{1}\right)=\left(\frac{d p_{1}}{d t}\right)^{2}=\left(I_{2}-p_{1}^{2}-\varepsilon\left(\sigma^{2}\right)\right)\left(2\left(H^{*}-p_{1}\right)-\frac{\sigma^{2}}{c_{1}}\right) \\
& -\left(I_{3}-p_{1} \sigma\right)^{2}-\varepsilon\left(\left(2 H^{*}-\frac{\sigma^{2}}{c_{1}}-2 p_{1}\right)^{2}\right)
\end{aligned}
$$

The function $f\left(p_{1}\right)$ is then a cubic function of $p_{1}$ and determines the qualitative behavior of an arbitrary elastic curve. The solutions are therefore given in terms of elliptic functions, as demonstrated in subsection III-A. $M_{2}$ and $M_{3}$ can be solved in terms of $p_{1}$ and the constants of motion. Proceeding to solve the extremals and using the Hamiltonian function (9) where $M_{1}$ is a constant $\sigma$, the reduced Hamiltonian is

$$
M_{2}^{2}+M_{3}^{2}=2\left(H^{*}-p_{1}\right)-\frac{\sigma^{2}}{c_{1}}
$$

This suggests using polar coordinates for $M_{2}$ and $M_{3}$;

$$
\begin{aligned}
& \theta(t)=\arctan \left(\frac{M_{2}}{M_{3}}\right) \\
& \dot{\theta}=\frac{M_{3} \dot{M}_{2}-M_{2} \dot{M}_{3}}{M_{2}^{2}+M_{3}^{2}}
\end{aligned}
$$

substituting in the values for $\dot{M}_{2}$ and $\dot{M}_{3}$ from (12) gives

$$
\begin{gathered}
\dot{\theta}=\frac{M_{3}\left(\frac{M_{1} M_{3}}{c_{1}}-M_{1} M_{3}+p_{3}\right)}{M_{2}^{2}+M_{3}^{2}} \\
+\frac{M_{2}\left(\frac{-M_{1} M_{2}}{c_{1}}+M_{1} M_{2}-p_{2}\right)}{M_{2}^{2}+M_{3}^{2}}
\end{gathered}
$$

and on simplifying can be expressed as:

$$
\dot{\theta}=\frac{\sigma}{c_{1}}-\sigma+\frac{I_{3}-p_{1} \sigma}{2\left(H^{*}-p_{1}\right)-\frac{\sigma^{2}}{c_{1}}}
$$

It is easy to solve explicitly for the radius from (19)

$$
r(t)=\sqrt{2\left(H^{*}-p_{1}\right)-\frac{\sigma^{2}}{c_{1}}}
$$

Therefore, the extremal control functions are

$$
\begin{aligned}
& u_{1}^{*}=\frac{M_{1}(t)}{c_{1}}=\frac{\sigma}{c_{1}} \\
& u_{2}^{*}=M_{2}(t)=r(t) \sin (\theta(t)) \\
& u_{3}^{*}=M_{3}(t)=r(t) \cos (\theta(t))
\end{aligned}
$$

Note that $\theta(t)$ is analogous to the nutation angle for the Lagrange top (see [16]). The extremals are therefore only dependent on the constants of motion and $p_{1}$.

\section{A. Explicit solution of $p_{1}$}

For the purpose of this paper the critical points of the cubic function (18) are of importance. However, we show here that (18) can be solved in terms of a Weierstrass' $\wp$-function, a meromorphic function of complex time $z$. The equations for $p_{1}$ will be converted into the canonical equation for the Weierstrass' $\wp$-function (see [17]):

$$
\dot{\wp}^{2}=4 \wp^{3}-g_{2} \wp-g_{3}
$$

where $g_{2}$ and $g_{3}$ are the elliptic invariants to be determined and that specify $\wp$ completely. The Weierstrass' $\wp$ function is often denoted $\wp\left(z ; g_{2}, g_{3}\right)$, where $z \in \mathbb{C}$. In this problem $z$ is time and therefore we restrict ourselves to the real values of $z$, then $\wp\left(z ; g_{2}, g_{3}\right)$ is real. The equation (18) can be converted into this canonical form via an affine input transformation:

$$
p_{1}=a \wp+b
$$

where $a$ and $b$ are constants and therefore

$$
\dot{p}_{1}=a \dot{\wp}
$$

Although the equation (18) can be solved for any constant $c_{1}$, we assume $c_{1}=1$ for simplicity of this illustration. Substituting (25) and (26) into (18) gives:

$$
\begin{aligned}
& a^{2} \dot{\wp}^{2}=2 a^{3} \wp^{3} \\
& +2 a^{2}\left(3 b-2 \varepsilon-H^{*}\right) \wp^{2} \\
& +2 a\left(3 b^{2}+4 \varepsilon H^{*}-2 b\left(2 \varepsilon+H^{*}\right)-I_{2}+I_{3} \sigma-\varepsilon \sigma^{2}\right) \wp \\
& +2 b^{3}-4 \varepsilon H^{* 2}-2 b^{2}\left(2 \varepsilon+H^{*}\right)+2 H^{*} I_{2} \\
& -I_{3}^{2}+2 \varepsilon H^{*} \sigma^{2}-I_{2} \sigma^{2}+2 b\left(4 \varepsilon H^{*}-I_{2}+I_{3} \sigma-\varepsilon \sigma^{2}\right)
\end{aligned}
$$

Comparing the coefficients of equation (27) to (24) and simplifying we obtain:

$$
\begin{aligned}
& a=2 \\
& b=\frac{2 \varepsilon+H^{*}}{3}
\end{aligned}
$$


Therefore, $p_{1}$ can be expressed explicitly as a Weierstrass' $\wp$-function under the affine transformation:

$$
p_{1}=2 \wp\left(z ; g_{2}, g_{3}\right)+\frac{2 \varepsilon+H^{*}}{3}
$$

Consequently $p_{1}$ is a meromorphic function and the elliptic invariants are:

$$
g_{2}=\frac{1}{3}\left(4 \varepsilon^{2}-8 \varepsilon H^{*}+H^{* 2}+3 I_{2}-3 I_{3} \sigma+3 \varepsilon \sigma^{2}\right)
$$

and

$$
\begin{aligned}
& g_{3}=\frac{1}{108}\left(32 \varepsilon^{3}+4 H^{* 3}-18 H^{*}\left(2 I_{2}+I_{3} \sigma\right)\right. \\
& +\varepsilon^{2}\left(-96 H^{*}+36 \sigma^{2}\right) \\
& \left.+12 \varepsilon\left(5 H^{* 2}+3 I_{2}-3 I_{3} \sigma-3 H^{*} \sigma^{2}\right)+27\left(I_{3}^{2}+I_{2} \sigma^{2}\right)\right)
\end{aligned}
$$

These calculations demonstrate that the integration procedure is essentially the same for all three space forms with the solutions only differing by the parameter $\varepsilon=1,-1,0$. In addition it is shown in [17] that the discriminant of the cubic $4 \wp^{3}-g_{2} \wp-g_{3}=0$ is

$$
\Delta=g_{2}^{3}-27 g_{3}^{2}
$$

and if $g_{2}$ and $g_{3}$ are real then the cubic $4 \wp^{3}-g_{2} \wp-g_{3}=0$, determines the qualitative nature of the solutions where the cubic has:

- three distinct real roots if $\Delta>0$.

- three real roots which are not all distinct if $\Delta=0$.

- a real root and a pair of complex conjugate roots if $\Delta<$ 0 .

\section{The SERRET-Frenet Frame: CURVATURE AND TORSION OF ELASTICA}

In order to draw geometric conclusions in terms of curvature and torsion of the corresponding elastic curves it is necessary to relate the extremals which have been solved to the curvature $\kappa$ and the torsion $\tau$ along the central line. Therefore, it is necessary to relate the differential equation (1) to the differential equation expressed in terms of curvature and torsion, called the Serret-Frenet frame:

$$
\frac{d \bar{g}(t)}{d t}=\bar{g}(t)\left(\begin{array}{cccc}
0 & -\varepsilon & 0 & 0 \\
1 & 0 & -\kappa & 0 \\
0 & \kappa & 0 & -\tau \\
0 & 0 & \tau & 0
\end{array}\right)
$$

where $\bar{g}(t) \in G$. Denote the standard basis elements of a vector in $\mathbb{R}^{4}$ as $\vec{e}_{1}, \vec{e}_{2}, \vec{e}_{3}, \vec{e}_{4}$ with $\bar{g}(t) \vec{e}_{1}=\gamma(t)$ where $\gamma(t)$ is a curve in the manifold $M$, then the tangent vector $\vec{T}=$ $\bar{g}(t) \vec{e}_{2}$, the normal vector $\vec{N}=\bar{g}(t) \vec{e}_{3}$ and the binormal vector $\vec{B}=\bar{g}(t) \vec{e}_{4}$. Therefore differentiating $\vec{T}$ and substituting in equation (29) gives:

$$
\begin{aligned}
& \frac{d \vec{T}}{d t}=\frac{d \bar{g}}{d t} \vec{e}_{2}=\bar{g}(t)\left(\begin{array}{cccc}
0 & -\varepsilon & 0 & 0 \\
1 & 0 & -\kappa & 0 \\
0 & \kappa & 0 & -\tau \\
0 & 0 & \tau & 0
\end{array}\right) \vec{e}_{2} \\
& =\bar{g}(t)\left(-\varepsilon \vec{e}_{1}+\kappa \vec{e}_{3}\right)=-\varepsilon \gamma(t)+\kappa \vec{N}
\end{aligned}
$$

and in the same manner as (30) obtain the differential equations describing the Serret-Frenet Frame:

$$
\begin{aligned}
& \frac{d \vec{N}}{d t}=-\kappa \vec{T}+\tau \vec{B} \\
& \frac{d \vec{B}}{d t}=-\tau \vec{N}
\end{aligned}
$$

Let $\vec{a}_{1}, \vec{a}_{2}, \vec{a}_{3}$ be an orthonormal frame fixed at a point on the body, called the moving frame. The Serret-Frenet Frame is shown to be explicitly related to the moving frame in [6]. Here we proceed to solve for the curvature and torsion of the elastic curves in terms of the meromorphic function $p_{1}$ and the constants of motion (9), (10), (11).

In these cases the Serret-Frenet frame and the elastic (general) frame are adapted to the curve $\gamma(t)$ in such a way that the first leg of the frame coincides with the tangent of the curve $\frac{d \gamma(t)}{d t}=\vec{a}_{1}=\vec{T}$ implies that $\vec{a}_{2}$ and $\vec{a}_{3}$ are in the normal plane spanned by $\vec{N}$ and $\vec{B}$. Define an angle $\beta$ that the normal $\vec{N}$ makes with $\vec{a}_{2}$ then:

$$
\begin{aligned}
& \vec{N}=(\cos \beta) \vec{a}_{2}+(\sin \beta) \vec{a}_{3} \\
& \vec{B}=(-\sin \beta) \vec{a}_{2}+(\cos \beta) \vec{a}_{3}
\end{aligned}
$$

Differentiating $\vec{N}$ gives

$\frac{d \vec{N}}{d t}=\frac{d \beta}{d t}\left((-\sin \beta) \vec{a}_{2}+(\cos \beta) \vec{a}_{3}\right)+(\cos \beta) \frac{d \vec{a}_{2}}{d t}+(\sin \beta) \frac{d \vec{a}_{3}}{d t}$

Let $R \in S O(3)$ be the matrix that relates coordinates in the moving frame to coordinates in the fixed frame along $\gamma(t)$, explicitly $\vec{a}_{i}=R \vec{e}_{i}$. In addition as the tangent space at $R$ can be either a left or right translation at the identity then $\frac{d R}{d t}=A(u) R$ where $A(u) \in \mathfrak{k}$ relates to the controlled vector fields (the horizontal vectors in the Cartan decomposition) in our problem:

$$
A(u)=\left(\begin{array}{ccc}
0 & -u_{3} & u_{2} \\
u_{3} & 0 & -u_{1} \\
-u_{2} & u_{1} & 0
\end{array}\right)
$$

and $\omega(t)$ is used to denote its corresponding coordinate vector related by $A(u) x=x \times \omega(t)$ for all $x \in \mathbb{R}^{3}$ where

$$
\omega(t)=\left(\begin{array}{l}
u_{1} \\
u_{2} \\
u_{3}
\end{array}\right)
$$

then

$$
\begin{aligned}
& \frac{d \vec{a}_{i}}{d t}=\frac{d R}{d t} \vec{e}_{i}=A(u) R \vec{e}_{i} \\
& =A(u) \vec{a}_{i}=\vec{a}_{i} \times \omega(t)
\end{aligned}
$$

from (34) and writing $\vec{a}_{i}$ in its standard basis we can obtain two useful equations:

$$
\begin{aligned}
& \frac{d \vec{a}_{2}}{d t}=-u_{3} \vec{a}_{1}+u_{1} \vec{a}_{3} \\
& \frac{d \vec{a}_{3}}{d t}=u_{2} \vec{a}_{1}-u_{1} \vec{a}_{2}
\end{aligned}
$$


substituting (35) into (33) and rearranging gives the following expression:

$$
\begin{aligned}
& \frac{d \vec{N}}{d t}=\left((-\cos \beta) u_{3}+(\sin \beta) u_{2}\right) \vec{a}_{1} \\
& -\left(\frac{d \beta}{d t} \sin \beta+u_{1} \sin \beta\right) \vec{a}_{2} \\
& +\left(\frac{d \beta}{d t} \cos \beta+u_{1} \cos \beta\right) \vec{a}_{3}
\end{aligned}
$$

Using the equation $\frac{d \vec{N}}{d t}=-\kappa \vec{T}+\tau \vec{B}$ from (31) and substituting in the expressions for $\vec{T}$ and $\vec{B}$ gives:

$$
\frac{d \vec{N}}{d t}=-\kappa \vec{a}_{1}+\tau\left((-\sin \beta) \vec{a}_{2}+(\cos \beta) \vec{a}_{3}\right)
$$

Identifying (36) with (37) yields:

$$
\begin{aligned}
& \kappa=u_{3} \cos \beta-u_{2} \sin \beta \\
& \tau=\frac{d \beta}{d t}+u_{1}
\end{aligned}
$$

To obtain a direct relation between the extremals and torsion it is necessary to obtain an expression for $\beta$ in terms of the controls. Differentiating $\vec{B}$ in (32) gives:

$$
\begin{aligned}
& \frac{d \vec{B}}{d t}=\frac{d \beta}{d t}\left((-\cos \beta) \vec{a}_{2}+(-\sin \beta) \vec{a}_{3}\right) \\
& +(-\sin \beta) \frac{d \vec{a}_{2}}{d t}+(\cos \beta) \frac{d \vec{a}_{3}}{d t}
\end{aligned}
$$

Substituting (35) into (39) gives:

$$
\begin{aligned}
& \frac{d \vec{B}}{d t}=\left(u_{2} \cos \beta+u_{3} \sin \beta\right) \vec{a}_{1} \\
& -\left(u_{1}+\frac{d \beta}{d t}\right)(\cos \beta) \vec{a}_{2}-\left(u_{1}+\frac{d \beta}{d t}\right)(\sin \beta) \vec{a}_{3}
\end{aligned}
$$

Substituting $\vec{N}$ from (32) into $\frac{d \vec{B}}{d t}=-\tau \vec{N}$ from (31) and equating to (40) immediately yields:

$$
u_{2} \cos \beta+u_{3} \sin \beta=0
$$

Thus the equation for the angle $\beta$ can be written explicitly in terms of the controls as:

$$
\tan \beta=-\frac{u_{2}}{u_{3}}
$$

Summarizing

$$
\begin{aligned}
& \kappa=u_{3} \cos \beta-u_{2} \sin \beta \\
& \tau=\frac{d \beta}{d t}+u_{1} \\
& \tan \beta=-\frac{u_{2}}{u_{3}}
\end{aligned}
$$

In our case the Hamiltonian corresponds to $H^{*}=p_{1}+\frac{1}{2}\left(\frac{\sigma^{2}}{c_{1}}+\right.$ $\left.M_{2}^{2}+M_{3}^{2}\right)$ which is generated by the optimal controls $u_{1}(t)=$ $\frac{M_{1}}{c_{1}}=\frac{\sigma}{c_{1}} u_{2}(t)=M_{2}$ and $u_{3}(t)=M_{3}$, therefore the curvature can be written explicitly in terms of the extremals. Squaring the equation for the curvature (43) and adding to the square of equation (41) yields:

$$
\kappa^{2}=u_{2}^{2}+u_{3}^{2}=M_{2}^{2}+M_{3}^{2}
$$

and substituting (9) into (44) gives:

$$
\kappa^{2}=2\left(H^{*}-p_{1}\right)-\frac{\sigma^{2}}{c_{1}}
$$

Here we proceed to solve the torsion in terms of the meromorphic function for this particular case. From (42) and substituting the extremals gives:

$$
\tan \beta=-\frac{M_{2}}{M_{3}}
$$

Differentiating (46) yields:

$$
\sec ^{2} \beta \frac{d \beta}{d t}=\frac{M_{2} \dot{M}_{3}-M_{3} \dot{M}_{2}}{M_{3}^{2}}
$$

substituting in the equations for $\dot{M}_{2}$ and $\dot{M}_{3}$ from (12) gives:

$$
\begin{aligned}
& \sec ^{2} \beta \frac{d \beta}{d t}=\frac{M_{2}\left(\frac{-M_{1} M_{2}}{c_{1}}+M_{1} M_{2}-p_{2}\right)}{M_{3}^{2}} \\
& -\frac{M_{3}\left(\frac{M_{1} M_{3}}{c_{1}}-M_{1} M_{3}+p_{3}\right)}{M_{3}^{2}}
\end{aligned}
$$

In addition using equation (46) to derive $\sec ^{2} \beta=\frac{M_{2}^{2}+M_{3}^{2}}{M_{3}^{2}}$ and substituting into (48) gives:

$$
\frac{d \beta}{d t}=M_{1}\left(1-\frac{1}{c_{1}}\right)-\frac{M_{2} p_{2}+M_{3} p_{3}}{M_{2}^{2}+M_{3}^{2}}
$$

then substituting (49) into the equation for the torsion (43) gives:

$$
\tau=-\frac{M_{2} p_{2}+M_{3} p_{3}}{M_{2}^{2}+M_{3}^{2}}+M_{1}
$$

using the constants of motion (16), recalling that $M_{1}=\sigma$ in the integrable case and equation (44), then (50) can be written in terms of the meromorphic function $p_{1}$ :

$$
\tau=-\frac{I_{3}-p_{1} \sigma}{\kappa^{2}}+\sigma
$$

As the equations (45) and (51) are expressed in terms of the constants of motion and the meromorphic function $p_{1}$, it is clear that if $p_{1}$ is constant then the curvature and torsion are also constant. Therefore, at the singularity the elastic curves have constant curvature and constant torsion and are therefore generalized helices. This implies that at the singularities of the cubic function (18) (when $\frac{d p_{1}}{d t}=0$ ), the projections onto the base space correspond to steady motions i.e. constant translations and/or constant rotations.

\section{THE GEOMETRY AT THE SINGULARITIES OF THE EXTREMAL CURVES}

In this section a discussion is given of the equilibria of the reduced Hamiltonian vector fields. From equations (12) it is clear to see that there exists a trivial fixed point in all the cases $(\varepsilon=-1,0,1)$ when $p_{1}=p_{2}=p_{3}=M_{1}=M_{2}=M_{3}=0$. At these fixed points the corresponding elastic curves are the geodesics. In addition a degeneracy occurs in the Euclidean case corresponding to the level set $I_{2}=0$. This is the case studied in [1] used to plan aeroplane landing trajectories in the 'Control Tower Landing Problem'. This degenerate result does not extend to the non-Euclidean cases. The analysis in 
[1] result from a highly simplified model where helical elastic curves are found under the condition that $I_{2}=0$. In this paper we study the general case when $I_{2} \neq 0$. In the general case there are equilibria of the system that are less obvious and can be seen from the explicit solutions which occur at the roots of the cubic $f\left(p_{1}\right)=0$ in (18). The equilibria of the reduced Hamiltonian system corresponding to the roots of this cubic will be called singularities. It is shown here that at the roots of the cubic the extremals define a closed periodic orbit. A geometric interpretation is given in terms of the intersection of invariant surfaces and explicit solutions stated. Here we begin with a brief comment on the level set $I_{2}=0$ before moving onto the singularities defined at the root of the cubic.

\section{A. The Level set $I_{2}=0$}

The level set $I_{2}=0$ (equation (10)) in the Euclidean case is analogous to the Euler top (left-invariant heavy top) in the mechanics literature (see [16]). $I_{2}=0$ when $\varepsilon=0$ implies that $p_{1}=p_{2}=p_{3}=0$ for all time. Along with the assumption $c_{1}=c_{2}=c_{3}$ which is the case studied in [1], the corresponding elastic curves were used to find optimal landing trajectories for airplanes, and where the equations of motion (12) degenerate to:

$$
\begin{aligned}
& \frac{d M_{1}}{d t}=0 \\
& \frac{d M_{2}}{d t}=0 \\
& \frac{d M_{3}}{d t}=0
\end{aligned}
$$

$M_{1}, M_{2}$ and $M_{3}$ are constants of motion and therefore the optimal controls are constants. In [1] it is shown that the elastic curves corresponding to constant controls $u_{i}$ are helices. In addition if $M_{1}$ is zero the elastic curves reduce to Riemannian circles. If all the controls are zero then the elastic curve are geodesics. In the spherical and hyperbolic case, this particular degeneracy does not occur. In these cases $I_{2}=0$ corresponds to $p_{1}=p_{2}=p_{3}=M_{1}=M_{2}=M_{3}=0$ for $\varepsilon=1$ where the elastic curves are geodesics and in the hyperbolic case $\varepsilon=-1$ implies that the extremals exist on the light-cone $p_{1}^{2}+p_{2}^{2}+p_{3}^{2}=M_{1}^{2}+M_{2}^{2}+M_{3}^{2}$. This case requires further investigation and is left to future research.

\section{B. Critical points of the cubic function $f\left(p_{1}\right)$}

The explicit solutions of the Hamiltonian vector fields are solely dependent on the constants of motion and the initial conditions of the meromorphic function $p_{1}$, which is defined in terms of elliptic functions. The initialization of $p_{1}$ is therefore essential in determining the qualitative behavior of the system. To understand the behavior of $p_{1}$ (equation (18)) we plot $\frac{d p_{1}}{d t}$ against $p_{1}$; this phase portrait is illustrated for the Euclidean case in Figure 1, the Spherical case in Figure 2 and the Hyperbolic case in Figure 3 with each isocline dependent on the constant $I_{2}$. The constants used were $M_{1}=\sigma=0.5, H^{*}=1, I_{3}=1$ and $I_{2}$ was set to different constants $1,2,3,4,6,9$ which correspond to different isoclines in the figures. Each of the three figures vary only by the parameter $\varepsilon$. For each value of $I_{2}$ there is a bounded component and an unbounded component, the smallest value of $I_{2}$ corresponds to the smallest bounded component and the right most unbounded component:

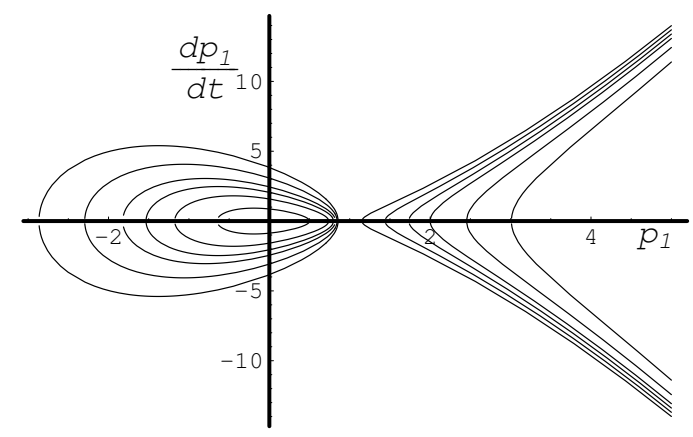

Fig. 1. Phase Portrait- the Euclidean case with increased $I_{2}$ the bounded component becomes larger and the unbounded component shifts to the left

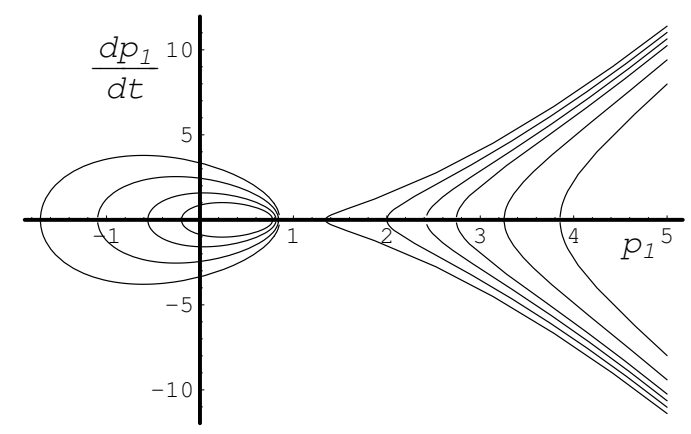

Fig. 2. Phase Portrait- the spherical case with increased $I_{2}$ the bounded component becomes larger and the unbounded component shifts to the left

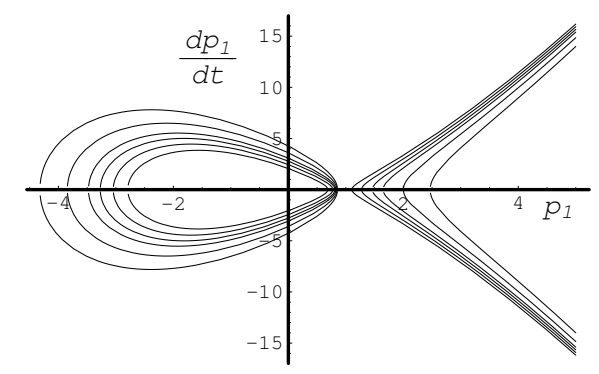

Fig. 3. Phase Portrait- the hyperbolic case with increased $I_{2}$ the bounded component becomes larger and the unbounded component shifts to the left

In the physical sense the bounded component is the only one that corresponds to real motions of the vehicle, as the extremals at these values of $p_{1}$ are real. In all three cases, provided the roots of the cubic are real the phase portraits are qualitatively unchanged. From the figures it is clear to see that $p_{1}$ is increasing above the horizontal axis $\frac{d p_{1}}{d t}>0$ and decreasing below the horizontal axis $\frac{d p_{1}}{d t}<0$. It is also clear to see that any initialization on the bounded component will flow to an equilibrium point i.e equilibrium points lie on the horizontal axis where $\frac{d p_{1}}{d t}=0$. It is only the isoclines that correspond to the unbounded component where $\frac{d p_{1}}{d t}>0$ that 
do not flow to an equilibrium point. Therefore considering only the possibility of real extremals, as $t \rightarrow \infty, p_{1}$ tends to a constant corresponding to a root of the cubic (18). In addition, as the curvature and torsion are both constant when $p_{1}$ is constant, the elastic curves corresponding to real extremals will flow to a helix as $t \rightarrow \infty$. Proceeding, assuming a constant $I_{2}$, we illustrate the different qualitative behavior of the critical points depending only on the curvature of the underlying space form. As $M_{1}$ is the constant $\sigma$ and can be initialized at any constant, a plot is given of the roots of the cubic equation $f\left(p_{1}\right)=0$, with $p_{1}$ a function of $\sigma$. In each of the following cases $H^{*}, I_{2}$ and $I_{3}$ were constant and only $\varepsilon$ was varied. A plot of the real roots/critical points are given in Figure 4 for $\varepsilon=0$, Figure 5 for $\varepsilon=1$ and Figure 6 for $\varepsilon=-1$ :

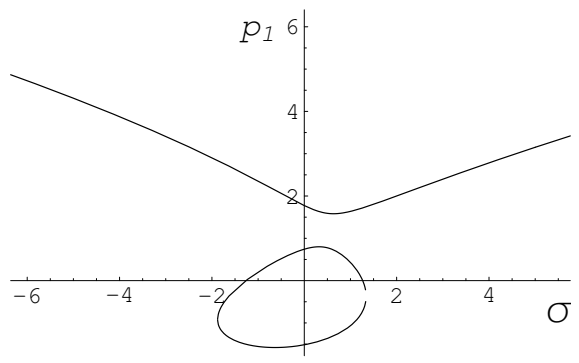

Fig. 4. The singularities of the system: Euclidean case $\varepsilon=0$

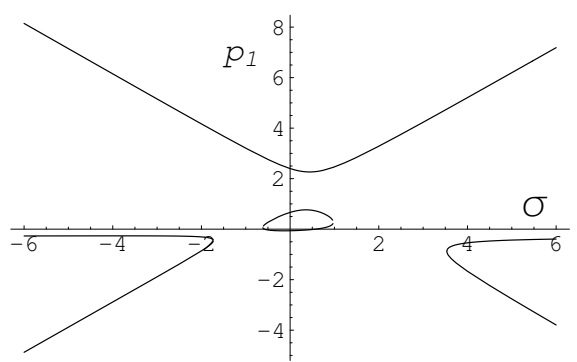

Fig. 5. The singularities of the system: Spherical case $\varepsilon=1$

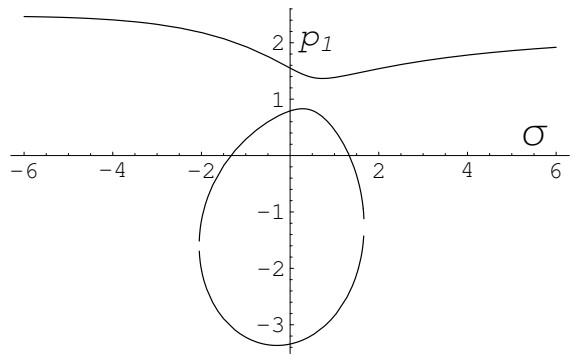

Fig. 6. The singularities of the system: Hyperbolic case $\varepsilon=-1$

The diagrams above give an indication in the differing qualitative nature of the solutions depending on the underlying space form.

\section{The geometry of the extremals at the singularity}

At the singularity defined at the critical points of the cubic, $p_{1}$ is constant and will be denoted by $c$. Then the Casimir functions (9), (10) and (11) can be written in a reduced form, see [4], where the left hand side of these equations are all constants:

$$
\begin{aligned}
& 2\left(H^{*}-c\right)-\frac{\sigma^{2}}{c_{1}}=M_{2}^{2}+M_{3}^{2} \\
& I_{2}-c^{2}-\varepsilon\left(\sigma^{2}\right)=p_{2}^{2}+p_{3}^{2}+\varepsilon\left(M_{2}^{2}+M_{3}^{2}\right) \\
& I_{3}-c \sigma=p_{2} M_{2}+p_{3} M_{3}
\end{aligned}
$$

writing the third equation in terms of $p_{2}$ and squaring gives:

$p_{2}^{2}=\frac{\left(I_{3}^{2}-2 I_{3} c \sigma+c^{2} \sigma^{2}\right)-2 I_{3} p_{3} M_{3}+2 c \sigma p_{3} M_{3}+p_{3}^{2} M_{3}^{2}}{M_{2}^{2}}$

defining new constants $\alpha=\left(I_{3}^{2}-2 I_{3} c \sigma+c^{2} \sigma^{2}\right)$ and $\gamma=$ $I_{2}-c^{2}-\varepsilon\left(\sigma^{2}\right)$ for simplicity, then by substituting $p_{2}^{2}$ into the second equation in (52), the reduced Hamiltonian and reduced Casimirs can be written as two surfaces in 3 dimensions $\left(p_{3}, M_{2}, M_{3}\right)$ :

$$
\begin{aligned}
& 2\left(H^{*}-c\right)-\frac{\sigma^{2}}{c_{1}}=M_{2}^{2}+M_{3}^{2} \\
& \gamma M_{2}^{2}=\alpha+p_{3}^{2} M_{2}^{2}+2 c \sigma p_{3} M_{3}-2 I_{3} p_{3} M_{3}+p_{3}^{2} M_{3}^{2} \\
& +\varepsilon M_{2}^{2}\left(2\left(H^{*}-c\right)-\frac{\sigma^{2}}{c_{1}}\right)
\end{aligned}
$$

Proceeding more geometrically we analyze the system at the singularities in terms of the intersection of these two invariant surfaces. For the purpose of the following illustration we take $\Delta>0$ where $\Delta$ is defined in equation (28) with positive reduced Hamiltonian i.e. the left hand side of the first equation in (52) is assumed positive, in a physical sense this is meaningful as energy is always positive. For each case the 3-dimensional surfaces are drawn graphically in Fig. 7. for $\varepsilon=0$, Fig. 8. for $\varepsilon=1$ and Fig. 9. for $\varepsilon=-1$.

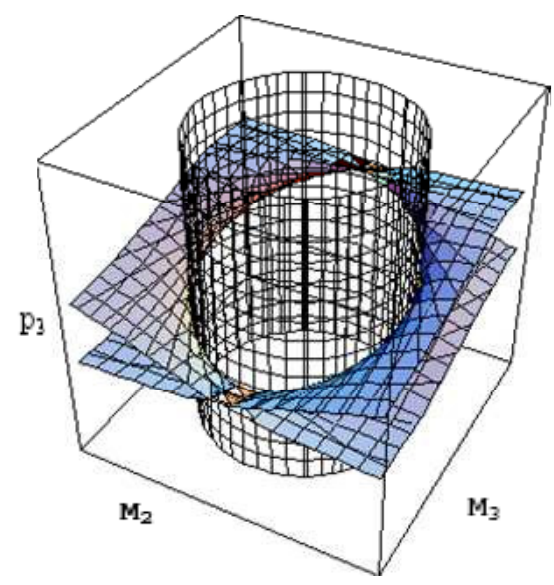

Fig. 7. The intersection of the energy cylinder and the non-generic quadric for $\varepsilon=0$ at the singularity 


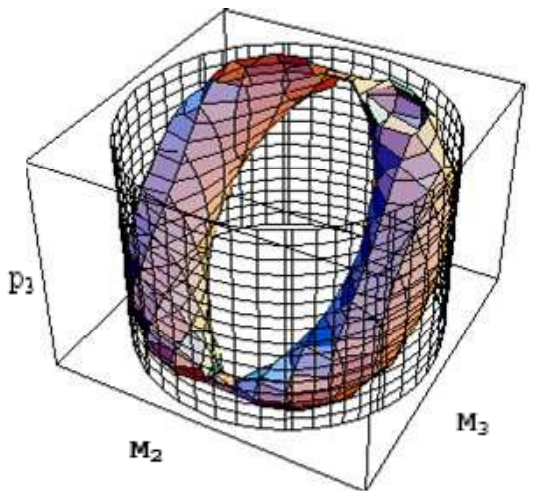

Fig. 8. The intersection of the energy cylinder and the non-generic quadric for $\varepsilon=1$ at the singularity

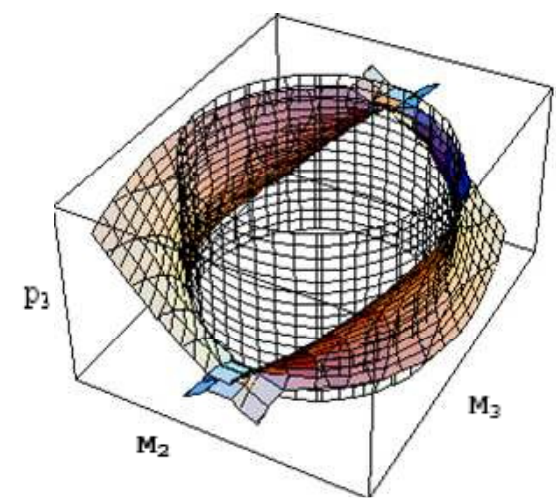

Fig. 9. The intersection of the energy cylinder and the non-generic quadric for $\varepsilon=-1$ at the singularity

The surfaces make contact and in each case the intersection is a closed periodic orbit. Fig. 10. shows the points of intersection for $\varepsilon=1$, this remains qualitatively unchanged in each case $\varepsilon=0$ and $\varepsilon=-1$.

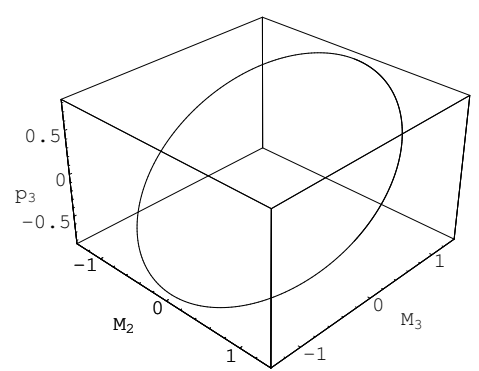

Fig. 10. Closed periodic orbit; intersection of invariant surfaces

When $p_{1}$ is constant, $M_{2}$ and $M_{3}$ are explicitly solved as (23), where $r$ is constant and $\theta$ is linear in $t$. Using the equation from (12)

$$
\frac{d M_{2}}{d t}=\frac{M_{1} M_{3}}{c_{1}}-M_{1} M_{3}+p_{3}
$$

and assuming $p_{1}$ is constant, we differentiate $M_{2}$ as solved in (23) and along with the solutions for $M_{1}, M_{2}, M_{3}$ are substituted in equation (54) and rearranged to give:

$$
p_{3}=\left(\sigma\left(\frac{c_{1}-1}{c_{1}}\right)+\dot{\theta}\right) r \cos (\theta(t))
$$

where $\dot{\theta}$ is a constant (21) when $p_{1}$ is constant. Therefore, in the frame $\left(p_{3}, M_{2}, M_{3}\right)$ the explicit solutions describe an ellipse as shown in Figure 10. To show this let us define a constant $\lambda=\left(\sigma\left(\frac{c_{1}-1}{c_{1}}\right)+\dot{\theta}\right) r$. Clearly, the projection onto the $M_{2}, M_{3}$ plane is a circle. In addition it is easily shown that the projection of this ellipse onto the $p_{3}, M_{2}$ plane is an ellipse from the explicit solutions and satisfy the implicit elliptic equation.

$$
\frac{p_{3}^{2}}{\lambda^{2}}+\frac{M_{2}^{2}}{r^{2}}=1
$$

Thus, the extremal curve is a circle when $\lambda=r$.

\section{The explicit solution of the periodic orbit}

Recall that assuming only real extremals as $t \rightarrow \infty, p_{1}$ tends to a constant defined by a root of the cubic (18). At these roots the solutions to the Hamiltonian vector fields simplify greatly. Immediately, from (23) where $r$ is constant and $\theta$ is linear in $t$ gives:

$$
\begin{aligned}
& M_{2}(t)=r \sin (\theta(t)) \\
& M_{3}(t)=r \cos (\theta(t))
\end{aligned}
$$

Then using the equations (12) to obtain the explicit expressions in the same manner as for equation (55) yields:

$$
\begin{aligned}
& p_{2}(t)=\lambda \sin (\theta(t)) \\
& p_{3}(t)=\lambda \cos (\theta(t))
\end{aligned}
$$

Therefore, at the critical points where $p_{1}=c$ is constant, the explicit solutions (57) and (58) define a closed periodic orbit in the plane $p_{1}, M_{1}$. From equation (21) with $p_{1}$ constant and assuming the initial value at $t=0$ to be $\theta_{0}=0$ then:

$$
\theta(t)=\left(\frac{\sigma}{c_{1}}-\sigma+\frac{I_{3}-p_{1} \sigma}{2\left(H^{*}-p_{1}\right)-\frac{\sigma^{2}}{c_{1}}}\right) t
$$

Equating $\theta$ to $2 n \pi$ where $n \in \mathbb{Z}$ the period $T$ of the closed orbit is:

$$
T=2 n \pi /\left(\frac{\sigma}{c_{1}}-\sigma+\frac{I_{3}-p_{1} \sigma}{2\left(H^{*}-p_{1}\right)-\frac{\sigma^{2}}{c_{1}}}\right)
$$

\section{E. The corresponding elastic curves at the singularity (Criti- cal Configurations)}

As $\kappa$ and $\tau$ are constant when $p_{1}$ is constant it is straightforward to integrate the equation (29) by taking the matrix exponential map from the Lie algebra to the Lie group (see [18]). An illustration of the different types of elastic curves that correspond to singularities in $\mathbb{E}^{3}$ is given. The illustration of $\mathbb{S}^{3}$ and $\mathbb{H}^{3}$ are omitted. The plot below shows a bifurcation diagram for the system for a particular set of constants. The critical points of $p_{1}$ are a function of the constant $I_{2}$, a plot of the real critical points are illustrated: 


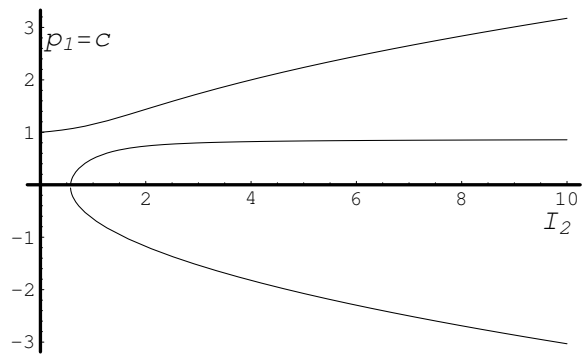

Fig. 11. Bifurcation diagram where the critical points of $p_{1}$ are dependent on $I_{2}$

As an illustration of the helical maneuvers of the vehicle we choose $I_{2}=2$ in Figure. 11. such that there are three real roots; a negative root, a small positive root and a larger positive root. For a fixed length of time, the vehicle traces the following elastic curve $\gamma(t)=g(t) \vec{e}_{1}=\left[x_{1}, x_{2}, x_{3}\right]^{T}$ :

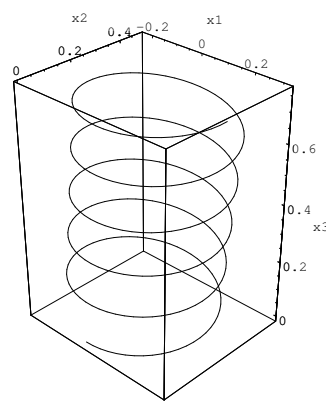

Fig. 12. Helix for $p_{1}=-1.17693$ and $I_{2}=2$, giving $\kappa=4.1$ and $\tau=0.406$

When $p_{1}=0.738226$ and $I_{2}=2, \kappa=0.27$ and $\tau=$ -7.93 . This is not illustrated here as for the same dimensions as Figure.12., the helix appears close to a straight line. Figure. 13. illustrates the helix for the large positive real root

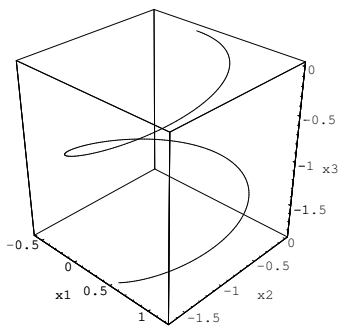

Fig. 13. Helix for $p_{1}=1.4387$ and $I_{2}=2$, giving $\kappa=-1.1275$ and $\tau=0.279$

Although the Serret-Frame gives a geometric interpretation of the elastic curves, it does not give any indication of how the vehicle rotates along these curves. For information about the rotation it is necessary to integrate the general frame (7). However, as the extremals are time dependent the integration procedure is not trivial and is the area of current research.

\section{CONCLUSION}

Oriented vehicles travelling at unit speed which are subject to steering controls that change their orientation can be modelled analogously to the elastic problem on Lie groups, where the Lie group describes the configuration space of the vehicle. The configuration spaces considered in this paper are the orthonormal frame bundles $S E(3), S O(4)$ and $S O(1,3)$. For these systems the extremal curves are solved explicitly in terms of elliptic functions. Under a transformation from the general frame to the Serret-Frenet frame, it is shown that the curvature and torsion of the corresponding elastic curves are also explicitly defined in terms of elliptic functions. In this paper an analysis at a singularity of these systems is given, defined at the roots of a cubic function that appear in the explicit solutions of the extremal curves. At these singularities it is shown that the extremal curves define a periodic orbit and the corresponding elastic curves have constant curvature and torsion. Therefore, the singularities in the extremal curves coincide with steady motions of the vehicle i.e. constant translation and/or constant rotation. Identifying such equilibria is extremely useful in the motion planning of vehicles. Using various stabilization techniques from geometric control such periodic equilibria can be exploited to obtain these steady motions. Although, the focus of this paper is on the motion control of vehicles travelling at unit speed, this paper presents new results applicable to general elastic curves and Kirchhoff's elastic rod. Future work will include a stability analysis of these singularities and methods to integrate the general frame in order to analyze the rotation of these vehicles as they trace elastic curves.

\section{REFERENCES}

[1] Walsh, G., Montgomery, R., Sastry, S., 'Optimal Path Planning on Matrix Lie Groups'. Proceedings of IEEE Conference on Decision and Control, 1994.

[2] Justh, E. W., Krishnaprasad, P. S., 'Natural frames and interacting particles in three dimension'. Proceedings of 44th IEEE Conference on Decision and Control and the European Control Conference, 2005.

[3] Leonard, N. E., Krishnaprasad, P. S., 'Motion control of drift-free, left-invariant systems on Lie groups'. IEEE Transactions on Automatic Control, 40(9):1539-1554, 1995.

[4] Biggs, J. D., Holderbaum, W., 'The geometry of optimal control problems on some Six dimensional Lie Groups'. Proceedings of 44th IEEE Conference on Decision and Control and the European Control Conference, 2005.

[5] Arroyo, J., Barros, M., Garay, O., 'Models of relativistic particles with curvature and torsion revisited'. General Relativity and Gravitation, Springer,Volume 36, Number 6, p.1441 - 1451, 2004.

[6] Jurdjevic, V., 'Geometric Control Theory'. Advanced Studies in Mathematics, Cambridge University Press, 52,1997.

[7] Jurdjevic, V., Monroy-Perez, F., 'Variational Problems on Lie Groups and their Homogeneous Spaces: Elastic Curves, Tops, and constrained Geodesic Problems' in Nonlinear Geometric Control Theory. World Scientific, 2002.

[8] Bloch, A. M., 'Nonholonomic Mechanics and Control'. Springer-Verlag, New York,2003.

[9] Crouch, P., Silva Leite, F., 'The Dynamic interpolation problem: On Riemannian manifolds, Lie groups and Symmetric spaces'. Journal of Dynamical and control systems, Vol.1, No. 2, p177-202, 1995.

[10] Bloch, A., Crouch, P., Ratui, T., 'Sub-Riemannian optimal control problems'. Fields Institute Communications, vol. 3, pp. 35-48, 1994.

[11] Hussein, I. I., Bloch, A. M., 'Optimal control of under-actuated systems with application to Lie Groups'. Proceedings of The American Control Conference, pp. 1472-1477, 2005. 
[12] Sussmann, H. J., 'An introduction to the coordinate-free Maximum Principle'. In Geometry of Feedback and Optimal Control,B. Jakubczyk and W. Respondek Eds., Marcel Dekker, New York, pp. 463-557, 1997.

[13] Jurdjevic, V., 'Integrable Hamiltonian Systems on Complex Lie Groups'. Memoirs of the American Mathematical Society, Vol. 178, No. 838, 2005.

[14] Ott, E., 'Chaos in Dynamical Systems'. Cambridge University Press, 1993.

[15] Goyal, S., Perkins, N. C., Lee, C. L. 'Nonlinear dynamics and loop formation in Kirchhoff rods with implications to the mechanics of DNA and cables'. Journal of Computational Physics, Vol. 209, Issue 1, pp. 371-389, 2005.

[16] Audin, M., 'Spinning Tops: A course on Integrable Systems'. Advanced Studies in Mathematics, Cambridge University Press, 1996.

[17] Lawden, D., 'Elliptic functions and applications'. Springer-Verlag, New York, 1989

[18] Hall, B. C., 'Lie Groups, Lie Algebras, and Representations: An Elementary Introduction'. Springer-Verlag, New York, 2003.

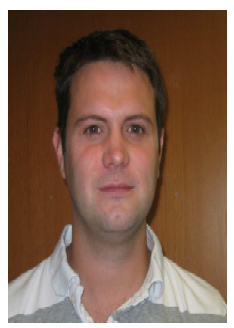

James Biggs received the B.Sc. degree in Mathematics with Economics from the University of Sussex, Brighton, UK in 1998 and the M.Sc. degree in Nonlinear Dynamics and Chaos from University College London, London, U.K. in 1999. Since then he has worked in Operational Research for the Defence Evaluation and Research Agency, U.K. He is currently a final year $\mathrm{PhD}$ student at the School of Systems Engineering, University of Reading, Reading, U.K. and his current research interests include geometric control theory and its applications.

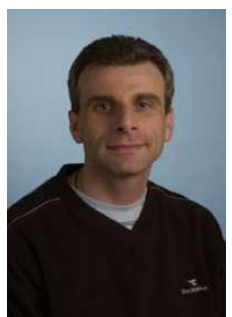

William Holderbaum received the M.Sc. degree in automatic control from the University of Reims, Reims, France 1993 and the $\mathrm{PhD}$ degree from the University of Lille, Lille, France 1999. He was a research assistant at the University of Glasgow, Glasgow, UK (1999-2001). He is currently a Lecturer in the School of Systems Engineering, University of Reading, Reading, U.K. His research interests are in control theory and its applications. These are mainly focused on boolean input systems (i.e., power converters), rehabilitation engineering (robust control design for unsupported paraplegic standing), and geometric control theory.

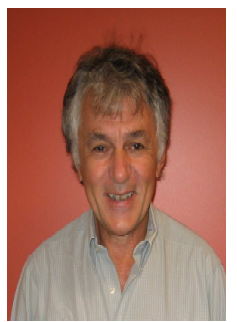

Velimir Jurdjevic received the B.Sc. degree in Electrical Engineering from Pennsylvania State University, M.Sc degree in Engineering and $\mathrm{PhD}$ degree in Mathematics both from Case Western Reserve University in Cleveland, Ohio. He was a research fellow at Harvard (1970-1971), upon which he took a post as a Professor of Mathematics at the University of Toronto. Currently he is Professor Emeritus at the same institution. His early research interests were in mathematical theory of accessibility and stability of non-linear control systems. Subsequently these interests shifted to optimal problems on Lie groups and their homogeneous spaces with applications to mechanics and geometry. Presently his research is focused on the role of symplectic geometry for problems of mathematical physics and quantum control. 